\title{
What do Prosumer Marginal Utility Functions Look Like? Derivation and Analysis
}

\author{
Ziras, Charalampos; Sousa, Tiago; Pinson, Pierre
}

Published in:

IEEE Transactions on Power Systems

Link to article, DOI:

10.1109/TPWRS.2021.3068620

Publication date:

2021

Document Version

Peer reviewed version

Link back to DTU Orbit

Citation (APA):

Ziras, C., Sousa, T., \& Pinson, P. (2021). What do Prosumer Marginal Utility Functions Look Like? Derivation and Analysis. IEEE Transactions on Power Systems, 36(5), 4322 - 4330.

https://doi.org/10.1109/TPWRS.2021.3068620

\section{General rights}

Copyright and moral rights for the publications made accessible in the public portal are retained by the authors and/or other copyright owners and it is a condition of accessing publications that users recognise and abide by the legal requirements associated with these rights.

- Users may download and print one copy of any publication from the public portal for the purpose of private study or research.

- You may not further distribute the material or use it for any profit-making activity or commercial gain

- You may freely distribute the URL identifying the publication in the public portal

If you believe that this document breaches copyright please contact us providing details, and we will remove access to the work immediately and investigate your claim. 


\title{
What do Prosumer Marginal Utility Functions Look Like? Derivation and Analysis
}

\author{
Charalampos Ziras, Member, IEEE, Tiago Sousa, Member, IEEE, Pierre Pinson, Fellow, IEEE
}

\begin{abstract}
Marginal utility functions (MUFs) encapsulate the prosumer willingness to trade energy with other market agents. In large-scale distributed optimization schemes scalability and convergence are crucial, and the assumption of linear MUFs is common. In this work, instead of assuming the shape and coefficients of those functions, a method is proposed to derive them based on the optimization of a prosumer's energy procurement problem. We formulate a rolling-horizon optimization problem that considers asset characteristics and network tariffs, and we utilize forecasts to capture the effects of uncertainty. We use this formulation to calculate the true prosumer MUF A test case with real data is used to investigate the shape of these functions. Our results reveal that they present a non-linear shape under certain conditions, they cannot be a priori derived, and their form is sensitive to a variety of factors. This indicates that MUFs should be constructed based on an approach similar to the one proposed in this paper to more accurately capture the prosumer's true willingness to trade. A linearized version of these functions, which is computationally attractive and scalable, can be determined using a least-squares estimator that achieves the best fit to the prosumer's preferences under a linearity constraint.
\end{abstract}

Index Terms-Energy management system, marginal utility function, peer-to-peer market, prosumer, stochastic optimization.

\section{INTRODUCTION}

\section{A. Background and motivation}

The decentralization of energy trading and asset coordination [1] are not new concepts in the field of power systems. They have been applied to a broad range of applications [2], from microgrid control [3] to ancillary service provision [4], collaborative demand response [5] and electricity market design $[\overline{6}]-[\overline{8}]$. Out of those applications, peer-to-peer (P2P) energy trading in particular has gained traction as a framework that is able to promote a more bottom-up operation of power systems through a more active role of small-scale prosumers at a residential level [9]. Prosumers are consumers who can also produce electricity, and are often enhanced with storage capabilities [10]. Current electricity markets have little room for active prosumers because existing regulation compels them to only procure energy from certified retailers [11]. Such market rules hinder the proactive behavior of prosumers, whereas $\mathrm{P} 2 \mathrm{P}$ trading can empower them with a higher degree of freedom on how they procure energy [12].

Different approaches can be followed to express a prosumer's willingness to engage in energy trading, depending on the application. The first is to solve a global optimization problem using all market participants' subproblems, usually via a distributed scheme to preserve privacy. The second is via bids of price/quantity, where a continuous double auction scheme optimally matches buyers and sellers [13]. The third, which is the focus of this work, is by replacing each prosumer's optimization problem with a marginal utility function (MUF) [14], [15]. This simplifies the representation of each market participant, providing a more suitable groundwork for both centralized and distributed trading schemes.

The first approach is more commonly found in small-scale applications, e.g., microgrids or energy trading in communities of limited size. The requirements of energy trading involving large numbers of participants pose limitations on the use of privacy-preserving negotiation processes based on each prosumer's optimization problem. For instance, each prosumer's problem may have a different structure and can involve integer variables or even be non-linear. In this case negotiation algorithms may fail to converge within a reasonable time frame (if they converge at all) when the number of participants is in the order of thousands, even if issues related to high-speed and reliable communication links are resolved.

These issues call for a simplified interface that can express the willingness of each prosumer to trade energy in a manner that is scalable and computationally manageable. As we show in the literature review, many works adopt linear MUFs [14], [15] because of their attractive properties for market clearing through distributed optimization algorithms (e.g., consensus alternating direction method of multipliers). Additionally, uncertainties, look-ahead actions, asset characteristics and end user constraints are most often neglected when deriving MUFs. and the parameters of those functions are arbitrarily chosen.

However, this generic approach may result in nonrepresentative MUFs because the prosumer's decision-making process is neglected. The authors of [16] recognize that several researchers focus on studying energy trading methods by relaxing, or even avoiding, the nature and characteristics of the participating assets. Adopting a simplistic approach in incorporating the physical dynamics of prosumers may lead to inefficient representation of their actual utility. We believe that a prosumer's true cost/benefit per procured energy unit must be calculated by considering the prosumer's operation, asset characteristics, future actions, the involved uncertainties and a proper forecast model. Further, those utility functions still have to retain certain properties, mainly that of convexity, to be applicable in various proposed $\mathrm{P} 2 \mathrm{P}$ market frameworks [17], [18]. It is thus interesting to see how realistic MUFs can be derived, if these satisfy these properties, and if an approximation is required to guarantee their convex shape. 

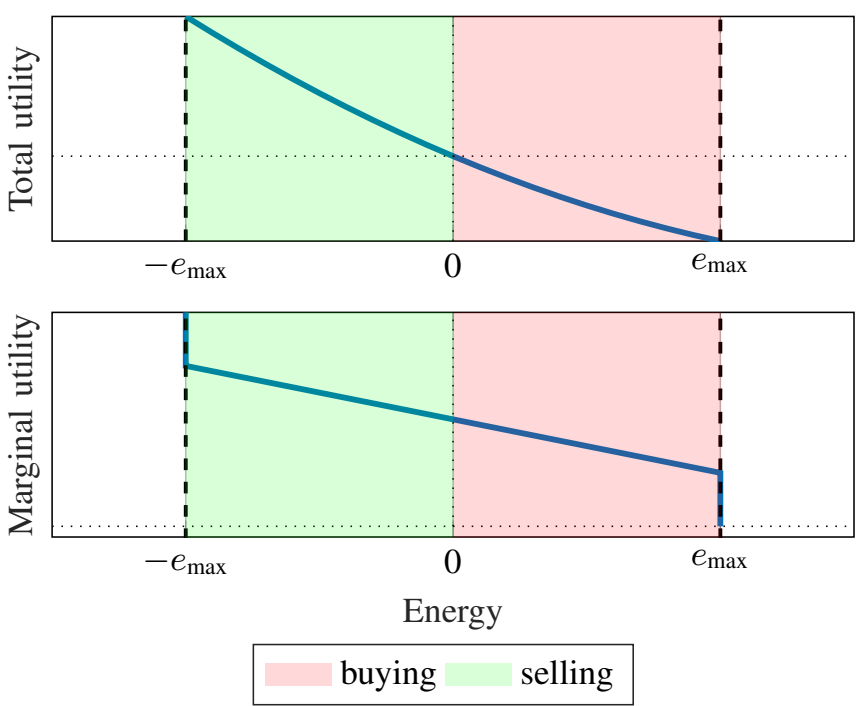

Fig. 1: Quadratic utility function (top) and linear MUF (bottom) for a prosumer.

\section{B. Literature review}

MUFs have a long history in power system applications [19] and their goal is to monetize the amount of energy consumed or produced by a prosumer [20]. In this section we focus on how such functions have been used in the context of energy trading and coordination, and how they are derived. An MUF expresses the maximum price per $\mathrm{kWh}$ of energy that a buyer is willing to pay and the minimum price value that a seller is willing to accept. The way that such functions are expressed and the used sign are often a matter of convention. We define utility as the revenue of a prosumer, making it positive (negative) when the prosumer sells (buys) an amount of energy. By convention, we define the energy bought by the prosumer as positive. In Fig. 1 an illustrative example based on [21] is shown. It depicts a quadratic utility function and the resulting linear MUF which is the most commonly used form in the literature. This means that the prosumer is willing to buy $e_{\max }$ even for lower prices than the corresponding endpoint; we have the reverse behavior for selling energy. In the remainder of the paper we will refer to linearity of the MUF in the range of $-e_{\max }$ and $e_{\max }$.

Paudel et al. [22] propose a Stackelberg game for $\mathrm{P} 2 \mathrm{P}$ trading, where participants are split into buyers and sellers, and quadratic functions are used to represent customer utility. Quadratic utility functions are also used in [21] for distributed multi-agent coordination in a microgrid. In both works the coefficients of the functions are arbitrarily chosen. In a similar fashion, Sorin et al. [17] introduce a $\mathrm{P2P}$ market structure based on a multi-bilateral economic dispatch formulation and also use quadratic utility functions with arbitrarily chosen parameters. Finally, Moret et al. [15] present a market structure for energy collectives where a non-profit virtual node (called the community manager) coordinates a set of prosumers. The prosumers' preferences, or else the coefficients of the used quadratic functions, are chosen in an arbitrary manner based on historical system prices and random sampling. Similar to these works, the authors of [23] use a piece-wise quadratic utility function for consumers and a quadratic function for producers, again with some set parameters. In [4] a $\mathrm{P} 2 \mathrm{P}$ setup for providing ancillary services by distributed energy resources is proposed, where the units' bids are also formulated with a linear MUF using fixed parameters.

The authors of [24] propose a Stackelberg game to capture the interactions between residential customers with renewable production and without individual storage and a shared facility controller. They express the utility of each customer by adding the natural logarithm of energy consumption (based on [25]) to the revenue from selling excess energy. Based on microeconomic theory, the authors of [25] proposed to use the natural logarithm of consumption (multiplied by a reference parameter) to express the utility of a prosumer. Similar to [24], the authors of [26] also propose a Stackelberg game to facilitate $\overline{\mathrm{P} 2 \mathrm{P}}$ trading of prosumers belonging to a microgrid, and model prosumer utility in the same manner. In [25] the preference parameters are arbitrarily set, and in [24] they are drawn from a uniform distribution.

\section{Research gap and contributions}

Our literature review shows that prosumer MUFs are most often modelled by assuming a type of function with desirable properties and then parameters are chosen in a largely arbitrary manner. For example, convexity is a necessary requirement [15], [27]. Sorin et al. require that prosumer functions must be convex and with a bijective gradient [17]. Baroche et al. (who also use quadratic cost functions) state that they must be closed, proper, and convex [18]. In general, quadratic utility functions, and thus linear MUFs are commonly used, largely because of their attractive properties [28]. Thus, the derived MUFs may not properly encapsulate the true marginal cost/benefit of the prosumer.

We believe that an optimization approach based on the prosumer's overall energy management problem is a more appropriate way of expressing utility when engaging in energy trading and for revealing the true marginal cost/benefit for the prosumer. Given the emergence of prosumer $\mathrm{P2P}$ trading [29], [30] and the support they receive from legislators, at least in the EU [12], in this work we focus on prosumers engaging in P2P trading. However, our approach of deriving MUFs is general and can be applied to other energy trading schemes as well. To this end, the contributions of this paper are threefold.

First, we review and discuss how MUFs are used in the literature to express the willingness of prosumers to trade energy. Second, we propose a methodology to derive a prosumer's MUF for an upcoming trading period by solving a stochastic optimization (SO) problem in a rolling horizon fashion, while considering uncertainties, future trades, prosumer constraints and asset characteristics, and incorporating a forecast model. It should be noted that the proposed methodology can also be used to derive price/quantity bids if the trading setup operates on such terms. Given that our derived MUFs can be nonlinear, the least-squares estimator is then used to retrieve a linear function, which is suitable for distributed optimization algorithms used in decentralized energy trading. The linearized 
function achieves a trade-off between employing each prosumer's full optimization problem, which is impractical for large-scale applications, and using a simplistic linear function that is arbitrarily parameterized. Third, we present a realistic test case and derive the respective MUFs, while providing a comprehensive analysis of how various factors such as tariffs, forecasting properties, and time of day affect those functions.

\section{Paper organization}

The remainder of this paper is organized as follows. After this introductory section, section II describes the prosumer's operation and interactions with the $\overline{\mathrm{P} 2 \mathrm{P}}$ market and explains how our methodology can be applied to derive MUFs Section III describes our test case. Section IV presents the derived MUFs under various conditions, discusses their properties and shows how they can be linearized. Section $\mathrm{V}$ concludes the paper.

\section{Problem formulation}

\section{A. Prosumer operation in a P2P market}

We consider prosumers who can procure energy via their retailers or via other prosumers through $\mathrm{P} 2 \mathrm{P}$ trading. Each prosumer can buy energy from the retailer at a price $\lambda_{t}^{\mathrm{b}}$ and sell at a price $\lambda_{t}^{\mathrm{s}}$ at each time step $t \in \mathcal{T}$, where $\mathcal{T}$ is the set for all time steps and has a cardinality of $|\mathcal{T}|=n_{\mathrm{T}}$. These prices differ from the spot prices to allow the retailer to make a profit. A fee $c_{t}$ is charged for energy imports, which relates to fees, network tariffs and taxes. The difference between the selling and buying prices results in a considerable price gap [31], especially due to the aforementioned fees [32].

The prosumer has an inflexible consumption $p_{t}^{1}$, which is expressed in energy terms. The duration of each time step $\Delta T$ is hourly-normalized, i.e., a $15 \mathrm{~min}$ step results in $\Delta T=0.25$. The prosumer is equipped with a non-controllable photovoltaic (PV) unit whose energy output is denoted by $p_{t}^{\mathrm{g}}$. Additionally, the prosumer owns a battery system with an energy capacity $\mathrm{C}$ (in $\mathrm{kWh}$ ), power capacity $\mathrm{P}^{\mathrm{N}}$ (in $\mathrm{kW}$ ) and efficiency $\eta$. It is straightforward to consider more flexible resources, such as electric vehicles (EVs) or thermal loads, without any fundamental modifications in our proposed methodology. However, for a clearer interpretation of our results we limit ourselves to one flexible resource. An overview of the data flows and interactions for the participation of the prosumer in the $\mathrm{P} 2 \mathrm{P}$ market is shown in Fig. 2

The prosumer has the option to trade energy in a $\mathrm{P} 2 \mathrm{P}$ fashion, instead of relying solely on the retailer for energy procurement. We consider a $\mathrm{P} 2 \mathrm{P}$ market where prosumers trade energy close to real-time solely for the upcoming time slot $t_{0}$, and not for multiple time steps. Each prosumer constructs an MUF for $t_{0}$ that is forwarded to the P2P market. After the market is cleared, each prosumer receives the market outcome. The prosumer settles any possible mismatches between the traded energy in the $\mathrm{P} 2 \mathrm{P}$ market and the physical power flows by procuring energy from the retailer ex-post.

Once the market outcome is communicated to the prosumer, an energy management system (EMS) controls the household's battery system. In practice, consumption and $\mathrm{PV}$ generation

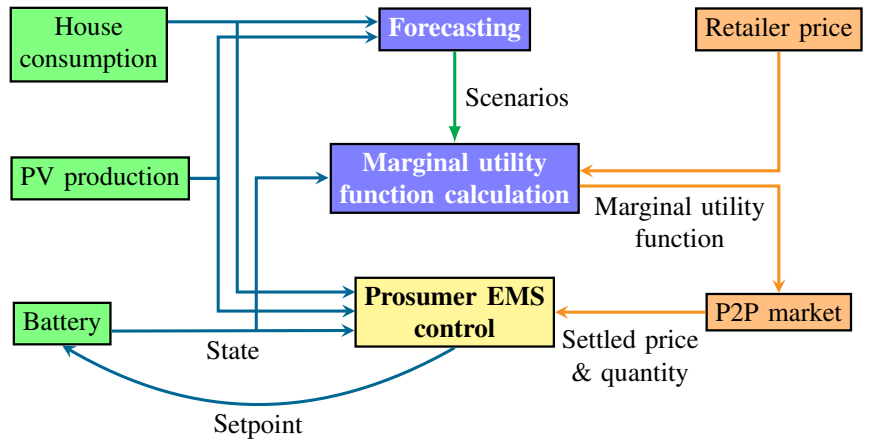

Fig. 2: Prosumer interactions in the $\mathrm{P} 2 \mathrm{P}$ market.

vary within the trading block period and the EMS could change the battery system's output within the trading interval. However, we consider only the exchange of energy and issues related to real-time control in faster time scales are out of the scope of this work.

These assumptions originate from the most commonly found framework in commercial $\mathrm{P2P}$ energy trading platforms, see [29], [30], as well as research proposals, see [33], [34]. According to current energy market regulations, prosumers do not fulfil the requirements for acting as a balance responsible party. For this reason they have to be associated with a retailer who takes that responsibility on their behalf. The alternative, where each prosumer assumes balance responsibility, would lead to an incompatible with the current regulations setup.

In this paper we focus on the MUF calculation functionality of the prosumer. The first block in Fig. 2 takes recent household consumption and $\mathrm{PV}$ production values and creates scenarios based on a forecasting model trained on past meter data. The second block creates an MUF by taking these scenarios, the battery's state and the retailer prices into account.

\section{B. Derivation of the MUF}

The MUF is generically formulated as $g\left(p^{\mathrm{p} 2 \mathrm{p}}\right) \doteq \lambda$ and expresses the amount of energy $p^{\mathrm{p} 2 \mathrm{p}}$ that the prosumer is willing to trade in the $\mathrm{P2P}$ market for different price values $\lambda$. The literature often assumes a convex shape for $g(\cdot)$. Alternatively, our paper presents a stochastic approach to reveal the shape of $g(\cdot)$ for an upcoming trading time step.

The MUF is derived through a set of pairs of values $\left(p_{n}^{\mathrm{p} 2 \mathrm{p}, *}, \lambda_{n}\right)$, with $n \in \mathcal{N}$. We treat $\lambda_{n}$ as a parameter which lies between the retailer selling price $\lambda_{t_{0}}^{\mathrm{s}}$ and buying price $\lambda_{t_{0}}^{\mathrm{b}}$. We define $\mathbb{P}(\lambda)$ as a set of optimization problems, parameterized by $\lambda$, where $\mathbb{P}$ formulates the prosumer's decision-making process. Then, $p_{n}^{\mathrm{p} 2 \mathrm{p}, *}:=\operatorname{argmin} \mathbb{P}\left(\lambda_{n}\right)$ reveals the amount of energy that the prosumer would trade for a price $\lambda_{n}$. The MUF is derived by repeating the process for all relevant $\lambda_{n}$ values.

$\mathbb{P}\left(\lambda_{n}\right)$ can be formulated as a multi-period optimization problem under uncertainty. Using a deterministic formulation instead has a significant effect on the shape of the MUF as we show later in section IV Different options, such as robust, chance-constrained or $\mathrm{SO}$ exist. A robust optimization formulation would be suitable in the case where it is necessary 
to satisfy some specific constraints even under the worst-case uncertainty realization. For example, those could be associated with guaranteeing a prosumer's thermal comfort if flexible thermal units are considered, or guaranteeing that a prosumer's EV is sufficiently charged by a given point in time. Other examples could include a power network constraint imposed on the prosumer which could limit the energy imports/exports. A chance-constrained formulation could be used to relax the above mentioned constraints if a low probability of violation is acceptable, thus making the prosumer's strategy less conservative. In our case a two-stage $\mathrm{SO}$ formulation is chosen because of the nature of the decision-making process. In the presented setup the prosumer has no need to satisfy any constraints as the ones described above, since we only consider a $\mathrm{PV}$ and a battery system and no network constraints exist. Our interest is to minimize the expected value of the prosumer's cost and $\mathrm{SO}$ is in most cases the most appropriate choice to do so.

At the first stage the optimal decision for the amount of energy that the prosumer is willing to trade for a set price $\lambda_{n}$ in the $\mathrm{P} 2 \mathrm{P}$ market is taken. At the second stage all the recourse decisions are taken depending on the uncertainty realization, with the goal of minimizing the expected prosumer costs. In this context, the objective function can be formulated as

$$
\min _{\mathcal{D}} \underbrace{f\left(p_{n}^{\mathrm{p} 2 \mathrm{p}}, \lambda_{n}\right)}_{\begin{array}{c}
\text { first stage } \\
\text { cost }
\end{array}}+\underbrace{\mathbb{E}_{w}\left[Q\left(p_{n}^{\mathrm{p} 2 \mathrm{p}}, \boldsymbol{\xi}\right)\right]}_{\begin{array}{c}
\text { recourse actions } \\
\text { cost }
\end{array}},
$$

subject to prosumer constraints which we describe later. $\mathcal{D}$ denotes the set of decision variables and $\boldsymbol{\xi}$ the data of the second-stage problem. By solving this optimization problem the optimal value of traded energy $p_{n}^{\mathrm{p} 2 \mathrm{p}, *}$ for $\lambda_{n}$ is obtained, resulting in a pair of values of the MUF. The idea here is that we bring all potential recourse costs to the first-stage decision through the objective function.

\section{Constraints}

After describing our methodology to derive the MUF below we formulate the $\mathrm{SO}$ problem in more detail. Both the inflexible load and the PV output are subject to uncertainty, which is expressed through scenarios $w \in \mathcal{W}$. Instead of separating the uncertainty of consumption and $\mathrm{PV}$ generation we express the uncertain net demand $p_{t, w}^{\mathrm{unc}}=p_{t, w}-p_{t, w}^{\mathrm{g}}$ as a single and time-dependent variable. The recourse actions are the scenario-dependent battery setpoints $\left(p_{t, w}^{\mathrm{c}}\right.$ for charging and $p_{t, w}^{\mathrm{d}}$ for discharging), the exchanges with the retailer ( $p_{t, w}^{\mathrm{b}}$ for buying and $p_{t, w}^{\mathrm{s}}$ for selling energy), and the future trades in the P2P market $p_{t, w}^{\mathrm{p} 2 \mathrm{p}}$. To enforce that $p_{t, w}^{\mathrm{c}}$ and $p_{t, w}^{\mathrm{d}}$ are mutually exclusive, a binary variable $d_{t, w}$ is introduced, such that

$$
\begin{array}{ll}
0 \leq p_{t, w}^{\mathrm{c}} \leq d_{t, w} \mathrm{P}^{\mathrm{N}} \Delta T, & \forall t \geq t_{0}, \forall w \\
0 \leq p_{t, w}^{\mathrm{d}} \leq\left(1-d_{t, w}\right) \mathrm{P}^{\mathrm{N}} \Delta T, & \forall t \geq t_{0}, \forall w .
\end{array}
$$

$X_{t}$ denotes the battery's state of charge (SOC) in $\mathrm{kWh}$. The following constraints describe the SOC evolution, enforce boundary conditions (starting and ending values $\mathrm{C}^{\mathrm{s}}$ and $\mathrm{C}^{\mathrm{e}}$, respectively), and limit the $\mathrm{SOC}$ in an interval between $10 \%$ and $100 \%$ of the energy capacity

$$
\begin{array}{lr}
X_{t, w}=X_{t-1, w}+p_{t, w}^{\mathrm{c}} \eta-p_{t, w}^{\mathrm{d}} / \eta, & \forall t \geq t_{0}, \forall w \\
X_{t_{0}-1, w}=\mathrm{C}^{\mathrm{s}}, \quad X_{n_{\mathrm{T}, w}}=\mathrm{C}^{\mathrm{e}}, & \forall w \\
0.1 \mathrm{C} \leq X_{t, w} \leq \mathrm{C}, & \forall t \geq t_{0}, \forall w .
\end{array}
$$

The balance between the traded energy and the physical energy flows must be respected for each scenario $w$. This balance is expressed as

$$
\begin{aligned}
& p_{n}^{\mathrm{p} 2 \mathrm{p}}+p_{t_{0}, w}^{\mathrm{b}}-p_{t_{0}, w}^{\mathrm{s}}=p_{t_{0}, w}^{\mathrm{unc}}+p_{t_{0}, w}^{\mathrm{c}}-p_{t_{0}, w}^{\mathrm{d}}, \quad \forall w . \\
& p_{t, w}^{\mathrm{p} 2 \mathrm{p}}+p_{t, w}^{\mathrm{b}}-p_{t, w}^{\mathrm{s}}=p_{t, w}^{\mathrm{unc}}+p_{t, w}^{\mathrm{c}}-p_{t, w}^{\mathrm{d}}, \quad \forall t>t_{0}, \forall w .
\end{aligned}
$$

Note that in (7) the $\mathrm{P} 2 \mathrm{P}$ traded energy $p_{n}^{\mathrm{p} 2 \mathrm{p}}$ at $t_{0}$ is the first-stage decision variable and thus not scenario-dependent. Conversely, future participation in the $\mathrm{P} 2 \mathrm{P}$ market for $t>t_{0}$ is expressed via recourse actions $p_{t, w}^{\mathrm{p} 2 \mathrm{p}}$ (see (8)). Variables $p_{t, w}^{\mathrm{b}}$ and $p_{t, w}^{\mathrm{s}}$ are mutually exclusive because the retailer buying price is strictly greater than the selling price, which avoids the inclusion of a binary variable. It is sufficient to constrain those variables in non-negative values as

$$
\begin{array}{ll}
p_{t, w}^{\mathrm{b}} \geq 0, & \forall t \geq t_{0}, \forall w \\
p_{t, w}^{\mathrm{s}} \geq 0, & \forall t \geq t_{0}, \forall w .
\end{array}
$$

Since only energy imports are subjected to tariff/tax payment, we introduce binary variables $g_{t, w}$, and the following constraints to distinguish the imported $x_{t, w}^{+}$from the exported $x_{t, w}^{-}$energy per scenario for each $t$. Using the big $M$ technique [35], this yields

$$
\begin{array}{ll}
p_{t, w}^{\mathrm{unc}}+p_{t, w}^{\mathrm{c}}-p_{t, w}^{\mathrm{d}}=x_{t, w}^{+}-x_{t, w}^{-}, & \forall t \geq t_{0}, \forall w \\
0 \leq x_{t, w}^{+} \leq g_{t, w} M, & \forall t \geq t_{0}, \forall w \\
0 \leq x_{t, w}^{-} \leq\left(1-g_{t, w}\right) M, & \forall t \geq t_{0}, \forall w
\end{array}
$$

A last set of constraints needs to be introduced to limit the energy that can be traded with the retailer to values within the range of possible physical energy flows. We made this assumption to avoid arbitrage by the prosumer between trades with other peers in the $\mathrm{P} 2 \mathrm{P}$ market and the retailer. In this way the prosumer uses the retailer only to settle any mismatches between the actual energy flows and the $\mathrm{P} 2 \mathrm{P}$ trades. This yields

$$
\begin{array}{ll}
p_{t, w}^{\mathrm{b}} \leq x_{t, w}^{+}, & \forall t \geq t_{0}, \forall w \\
p_{t, w}^{\mathrm{s}} \leq x_{t, w}^{-}, & \forall t \geq t_{0}, \forall w .
\end{array}
$$

\section{Objective function}

The objective function consists of two terms and for a given $\lambda_{n}$ the prosumer's optimization problem is formulated as follows:

$$
\begin{array}{ll}
\min _{\mathcal{D}} & \lambda_{n} p_{n}^{\mathrm{p} 2 \mathrm{p}}+\mathbb{E}_{w}\left[\sum_{t \in \mathcal{T}}\left(p_{t, w}^{\mathrm{b}} \lambda_{t}^{\mathrm{b}}-p_{t, w}^{\mathrm{s}} \lambda_{t}^{\mathrm{s}}+c_{t} x_{t, w}^{+}\right)\right] \\
& +\mathbb{E}_{w}\left[\sum_{t \in \mathcal{T} \backslash t_{0}} p_{t, w}^{\mathrm{p} 2 \mathrm{p}} \tilde{\lambda}_{t, w}\right],
\end{array}
$$

with the following set of decision variables $\mathcal{D}=$ $\left\{\left(p_{n}^{\mathrm{p} 2 \mathrm{p}}, p_{t, w}^{\mathrm{p} 2 \mathrm{p}} \in \mathbb{R}\right),\left(p_{t, w}^{\mathrm{c}}, p_{t, w}^{\mathrm{d}}, p_{t, w}^{\mathrm{b}}, p_{t, w}^{\mathrm{s}}, X_{t, w}, x_{t, w}^{+}, x_{t, w}^{-}\right) \in\right.$ 


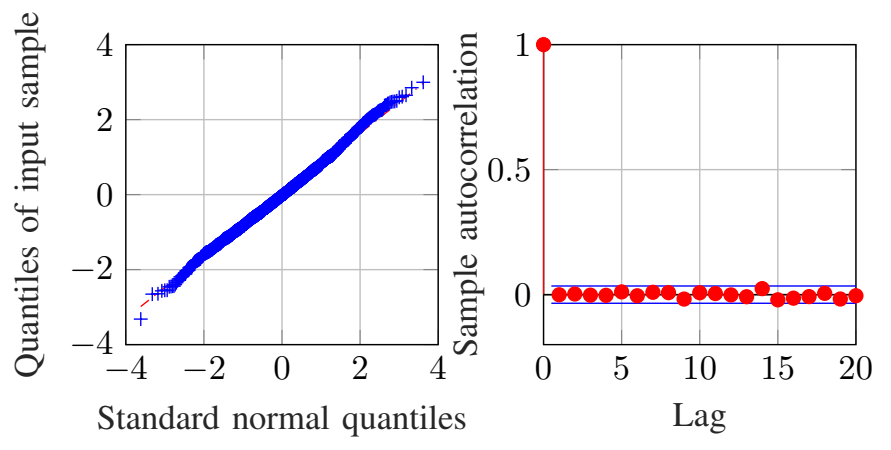

Fig. 3: Left plot: QQ plot of the residuals. Right plot: autocorrelation of the residuals.

$\mathbb{R}_{\geq 0},\left(d_{t, w}, g_{t, w} \in\{0,1\}\right\}_{n \in \mathcal{N}}$, and subject to constraints (2)(15). The first term corresponds to the prosumer's cost from $\mathrm{P} 2 \mathrm{P}$ trading in the upcoming period $t_{0}$. The other two terms express the expectation of the scenario-dependent costs, or else the recourse actions cost. More specifically, the second term includes the cost from procuring energy from the retailer and possible tariff payments for energy imports. The final term includes the cost from trading in the $\mathrm{P} 2 \mathrm{P}$ market for time steps $t>t_{0}$. Prices $\tilde{\lambda}_{t, w}$ are the future unknown cleared prices in the $\mathrm{P} 2 \mathrm{P}$ market.

\section{Case Study Description}

We use a real dataset from households in Australia which is shared as open data [36]. Households have their PV]production and both their flexible and inflexible load metered separately. The resolution is equal to 30 minutes and thus $\Delta T=0.5$. In the original dataset most households have a larger consumption than generation. It is more interesting to study customers who have an accumulated net demand that is close to zero over a day, so that they are largely self-sufficient and have more degrees of freedom when they utilize their flexibility. To this end, we chose a random customer for whom we neglect the flexible load and double the PV output. Various forecasting techniques can be used to obtain scenarios for a prosumer's future net demand. The goal of this paper is not to propose a new forecasting technique, but rather show how forecasting can be incorporated in the prosumer's derivation of the MUF. For the purpose of the paper we use a generic method without external regressors.

We use ten weeks as a training dataset and we perform a transformation because of the non-stationarity of the data. First, data is split into 48 sub-time series, one for each time step. Second, differencing is applied to remove any underlying trends. Third, the cumulative distribution function (CDF) of each sub-time series is calculated and data points are mapped to an interval between zero and one. Fourth, we apply a transformation of those data points by applying the inverse standard normal CDF Finally, the sub-time series are assembled to one time series and an ARMA model is fitted. Note that simulations will produce scenarios for the transformed data and the inverse procedure must be followed to obtain scenarios for the actual values.
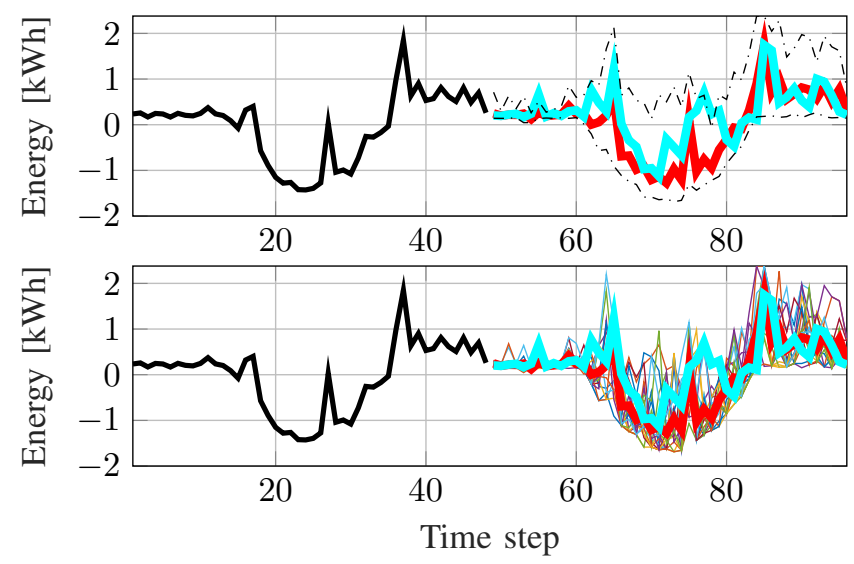

forecast $\longrightarrow$ past values $=$ realization.$--.95 \%$ PI

Fig. 4: Upper plot: Net demand forecast and 95\% prediction interval (PI) Lower plot: 20 net demand scenarios.

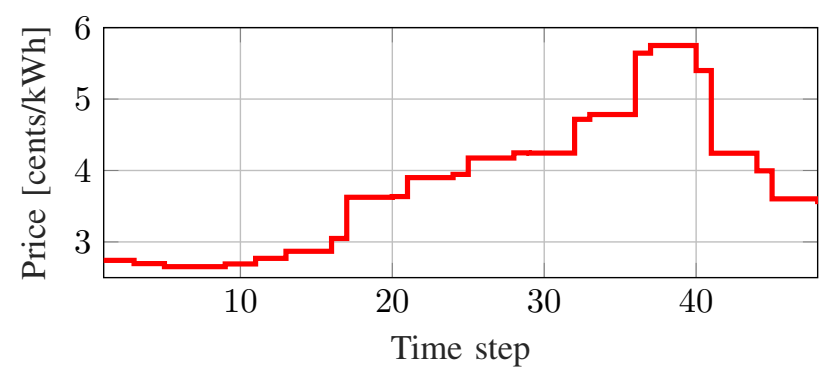

Fig. 5: Spot prices used for the case study.

An ARMA(3,4) model was chosen based on the AIC criterion and fitted on the transformed training dataset. In Fig. 3 the QQ plot of the residuals shows that these are normally distributed, without any significant autocorrelation. An example of such a forecast is shown in Fig. 4 for a horizon of 48 time steps (one day). The proposed method is studied for a single prosumer, where we use typical spot prices of the Danish power system, a flat tariff $c=0.2 € / \mathrm{kWh}$ and battery parameters $\eta=0.95, \mathrm{C}=5 \mathrm{kWh}, \mathrm{P}^{\mathrm{N}}=3 \mathrm{~kW}$. The spot prices used in this case study are shown in Fig. 5. Uncertainty set $\mathcal{W}$ consists of 100 equiprobable scenarios, generated at each required time step via the described ARMA model.

The future prices $\tilde{\lambda}_{t, w}$ in the $\mathrm{P} 2 \mathrm{P}$ market are in principle unknown and subject to uncertainty. Since we have no historical data regarding the operation of the market, we set all $\tilde{\lambda}_{t, w}$ for $t>t_{0}$ equal to the spot prices. If historical prices exist, a forecasting model can be used to create scenario-dependent $\tilde{\lambda}_{t, w}$. It should also be noted that retailer prices and $\mathrm{P} 2 \mathrm{P}$ prices $\tilde{\lambda}_{t, w}$ can be tweaked to express user preferences. This can be done by adjusting these prices depending on certain features (proximity, $\mathrm{CO}_{2}$ emissions etc.), which would result in different prices perceived by the prosumer. However, doing so would again require historical and behavioral data from the operation of the prosumer in a $\mathrm{P} 2 \mathrm{P}$ market under such terms. 
(a) $t_{0}=2$

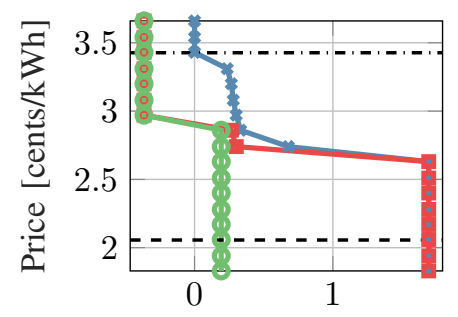

(c) $t_{0}=17$

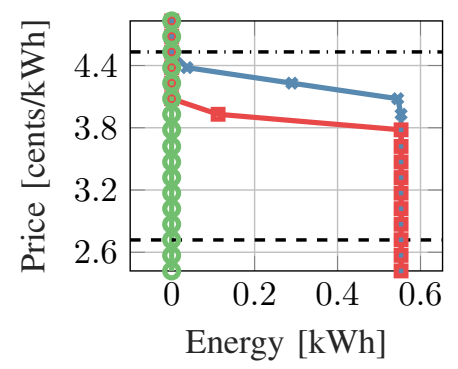

(b) $t_{0}=9$

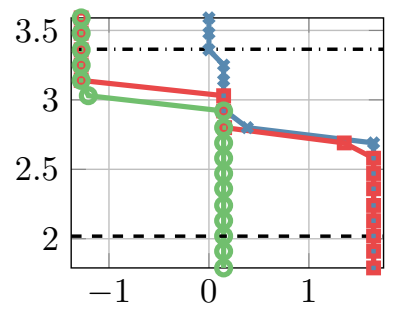

(d) $t_{0}=36$

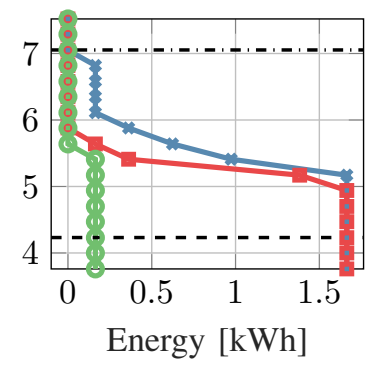

$0.1 \mathrm{C}=0.5 \mathrm{C}-\mathrm{C}---\lambda_{t_{0}}^{\mathrm{s}}-\cdots \lambda_{t_{0}}^{\mathrm{b}}$

Fig. 6: MUFs for four time steps and varying initial SOC values when tariffs are neglected.

\section{RESUlts}

\section{A. Effect of time step and initial SOC}

The prosumer constructs an MUF at each time step to trade energy in the upcoming trading period, which is then forwarded to the market. The shape of the function depends on the exact time step $t_{0}$ that it is constructed and the initial SOC of the battery $\left(X_{t_{0}-1}\right)$. Even though an MUF is created at each time step, this incorporates all future prosumer actions (future $\mathrm{P} 2 \mathrm{P}$ trades, exchanges with the retailer and use of the battery system) with the goal of minimizing energy costs on expectation. The MUFs for four different time steps and three varying levels of initial SOC are shown in Fig. 6 and Fig. 7. when tariffs are neglected and applied, respectively. The initial SOC plays an important role in the derived functions. Starting with Fig. 6, the prosumer is willing to buy less (or sell more) energy for a given price as the initial SOC value increases. This is an expected behavior, but the exact effect on the MUF is not always the same.

The existence of tariffs has a profound effect on the shape of the MUFs By comparing Fig. 6 and Fig. 7) it can be seen that the prosumer is less willing to buy energy in the $\mathrm{P} 2 \mathrm{P}$ market when tariffs are included because arbitrage is less profitable. In the considered cases the prosumer is not willing to sell energy at any price, even when the battery is fully charged. This happens because if there is insufficient $\mathrm{PV}$ production at a later period the prosumer would need to import energy at a very high cost. This leads to a different overall trading strategy, where the prosumer is willing to buy only small amounts of energy, unless the price is significantly lower than the spot price or the $\mathrm{SOC}$ is at low levels (e.g. at $t_{0}=2$ or $t_{0}=36$ ).

(a) $t_{0}=2$

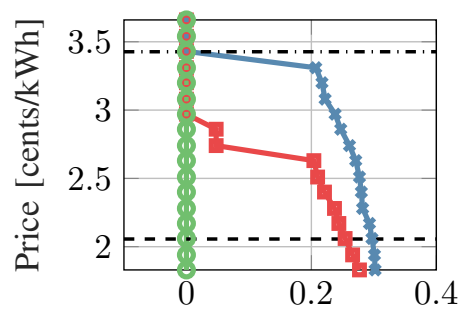

(c) $t_{0}=17$
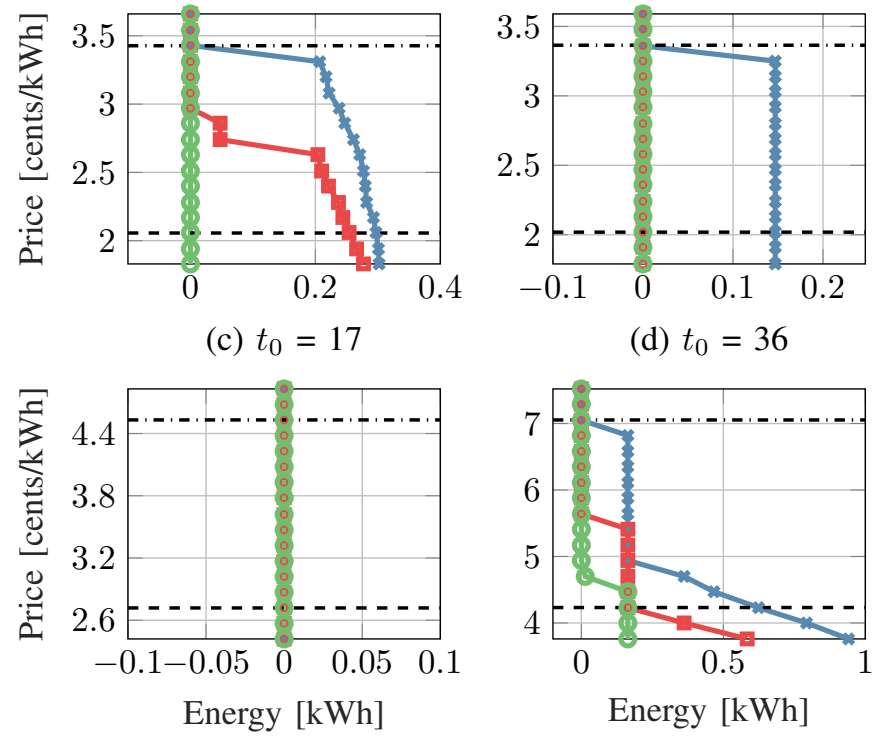

(d) $t_{0}=36$

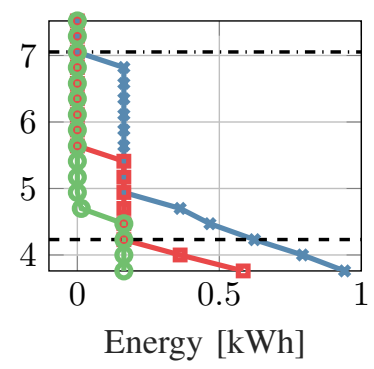

$-0.1 \mathrm{C} \rightarrow-0.5 \mathrm{C}-\mathrm{C}-\cdots \lambda_{t_{0}}^{\mathrm{s}} \cdots \cdots \lambda_{t_{0}}^{\mathrm{b}}$

Fig. 7: MUFs for four time steps and varying initial SOC values when tariffs are applied.

\section{B. Effect of the used uncertainty set}

In the previously derived functions 100 scenarios were used to represent $\mathcal{W}$. In this subsection the effect of different representations of uncertainty is examined by deriving an MUF while using 5, 10, 25 and 50 randomly chosen scenarios. Since the result is affected by the choice of those scenarios, the process is repeated 100 times for each case and the results are then described with probabilistic terms. We compare these results with the functions derived by using 100 scenarios and a deterministic case where one scenario with the expected net demand is used. The results for $t_{0}=2$, an initial SOC value of $0.5 \mathrm{C}$ and without employing tariffs are shown in Fig. 8 The blue curves are derived by using the median values from the 100 simulations. The light blue area indicates the $\pm 2 \sigma$ of the resulting energy values. Three interesting observations can be made.

First, as the number of used scenarios increases, on expectation the prosumer is willing to sell less energy in the market for the same $\lambda_{n}$. In other words, as the number of scenarios increases the prosumer tends to become more conservative and trades less energy $p_{n}^{\mathrm{p} 2 \mathrm{p}}$. This difference is most evident when the deterministic MUF and the one obtained with 100 scenarios are compared. This can be explained by looking at (7)-(8) and (14)-(15). Including more scenarios, especially those on the tail of the distribution of the uncertainty set $\mathcal{W}$, restricts the feasible region of $p_{n}^{\mathrm{p} 2 \mathrm{p}}$. Thus, the prosumer trades less energy in the $\mathrm{P} 2 \mathrm{P}$ market, so that it is still possible to cover any mismatch (see balance constraint (7)) due to the realization of the uncertain net demand.

Second, the variance of the resulting MUF decreases as the number of scenarios becomes larger. This is attributed to the 
(a) 5 scenarios

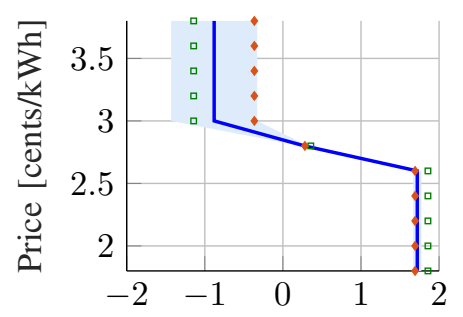

(c) 25 scenarios

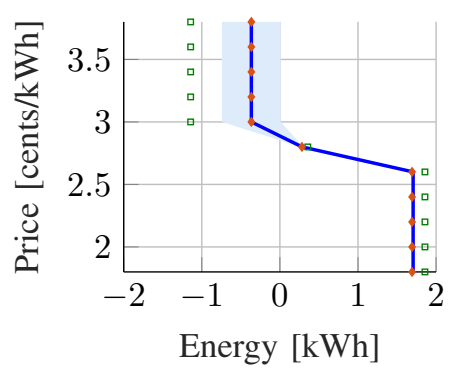

$\square$
$\square$ deterministic case $\quad 100$ scenarios

Fig. 8: MUFs for different uncertainty representations.

significant impact that few extreme scenarios can have in the optimization problem when the total number of scenarios is small. Such an effect can be seen when we consider only 5 scenarios, where the probability of each scenario $w$ will be equal to 0.2 . As a result, when the number of scenarios increases from 5 to 50 , the variance of the MUF reduces approximately threefold.

Third, the variance is significantly larger for selling energy $p_{n}^{\mathrm{p} 2 \mathrm{p}}$, compared to buying. This is mainly attributed to the point in time when the MUF is derived. During the night (which is the case for $t_{0}=2$ ), the prosumer needs to consume energy. Therefore, it is always beneficial to purchase energy in the $\mathrm{P} 2 \mathrm{P}$ market when prices are very low. This is not necessarily the case when the prosumer decides to sell energy at this same time step $t_{0}=2$. Doing so at this point in time may translate in extra benefits only if the realized accumulated demand until the time where the $\mathrm{PV}$ system starts producing energy is not very high. In the opposite situation when the realized demand is relatively high, the prosumer will need to buy energy at higher retailer prices later on. This uncertainty in the economic performance leads to the significantly larger variance in selling energy in the $\mathrm{P} 2 \mathrm{P}$ market.

\section{Comparison with benchmark}

The most common approach in the literature is to assume quadratic utility functions, as in [15], [21], resulting in linear MUFs In these works each asset class (i.e., inflexible load, PVs or batteries) is assumed to interact independently with the market and has its own function. However, in the majority of cases it is hard to assume an MUF per asset due to technical and regulatory issues, e.g., a consumer has a single connection

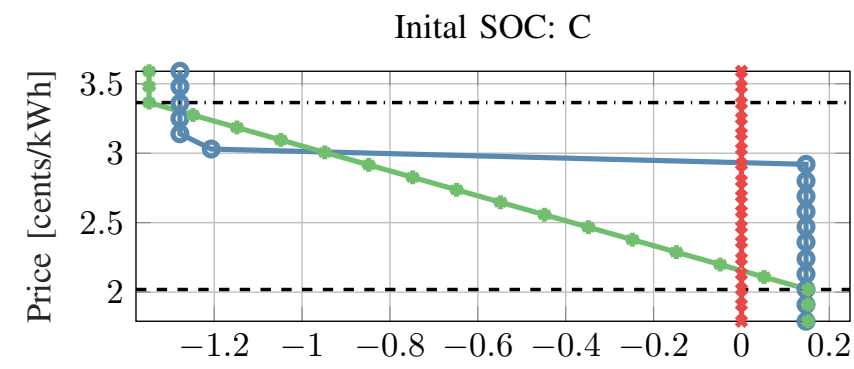

Initial SOC: $0.5 \mathrm{C}$
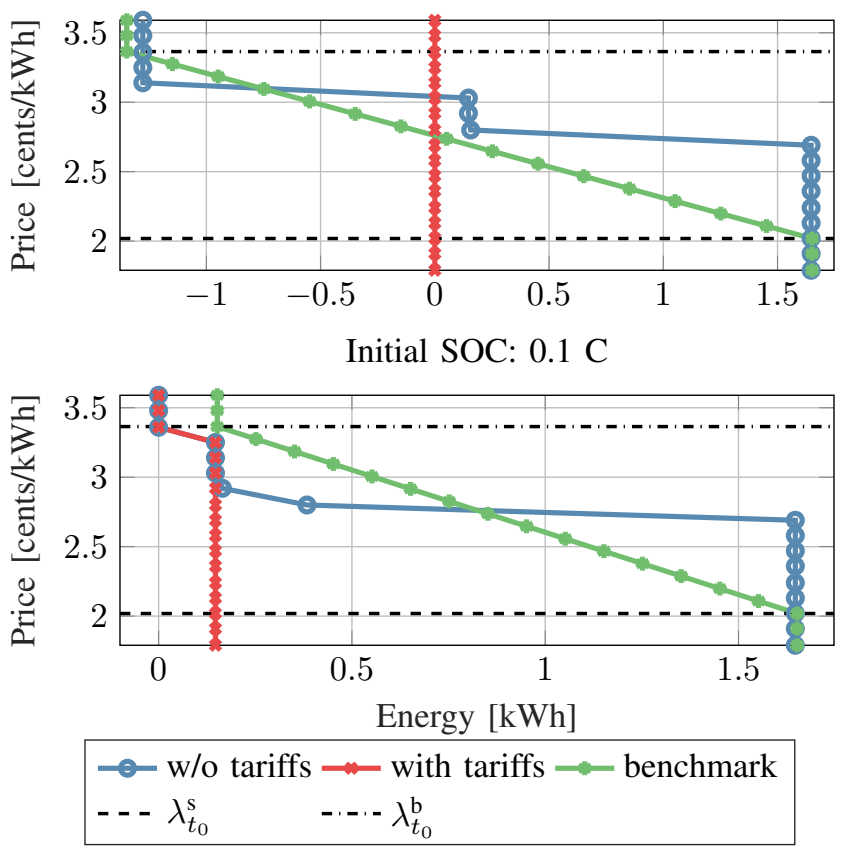

Fig. 9: Comparison between the derived functions with a linear MUF for $t_{0}=9$ and various initial $\mathrm{SOC}$ values.

agreement and a certified smart meter per asset usually does not exist. In this work we construct one function per prosumer, considering the total exchanged energy in the $\mathrm{P} 2 \mathrm{P}$ market and using the same convention as previously (positive refers to import of energy from the prosumer's perspective).

We construct linear MUFs which we will refer to as the benchmark in the following manner. First, the expected net demand $p_{t, w}^{\mathrm{unc}}$ for the upcoming trading period $t=t_{0}$ is calculated. Second, the allowed range of energy imports and exports is calculated by adding the maximum possible charging/discharging energy $\left(p_{t, w}^{\mathrm{c}}, p_{t, w}^{\mathrm{d}}\right)$ to the expected net demand, taking the initial SOC into consideration. Third, a linear function is derived by considering two points: the maximum imported energy for the retailer sell price $\lambda_{t_{0}}^{\mathrm{s}}$, and the maximum exported energy for the retailer buy price $\lambda_{t_{0}}^{\mathrm{b}}$. A comparison between these benchmark linear functions and the ones calculated by the proposed methodology for $t_{0}=9$ are shown in Fig. 9

The value of traded energy $p^{\mathrm{p} 2 \mathrm{p}}$ may be constant for a wide range of prices, but it can change drastically over minor differences in price $\lambda$. This can be observed for example in the middle subplot, where $p^{\mathrm{p} 2 \mathrm{p}}$ is equal to $1.65 \mathrm{kWh}$ for 


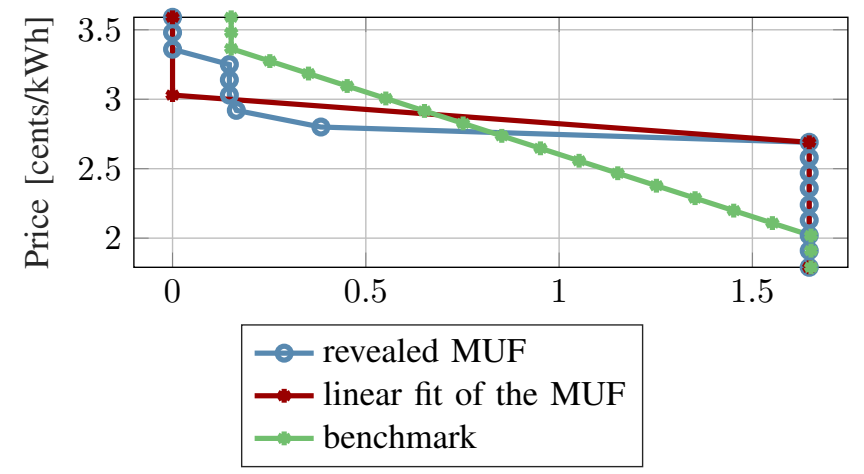

Fig. 10: Linearized fit of the MUF for the bottom plot of Fig. 9 when tariffs are excluded.

prices between 1.8 and 2.7 cents, decreasing to $0.15 \mathrm{kWh}$ for prices between 2.8 and 3 cents, and becoming negative $(-1.28 \mathrm{kWh})$ for prices above 3.1 cents. This behavior cannot be captured by the benchmark approach, because even if a linear fit could be a good approximation, the exact price range of the approximately linear part (2.7-3.1 cents) can only be revealed by an optimization-based approach. The benchmark approach performs significantly worse with the inclusion of tariffs. This happens because this simple approach does not have look-ahead considerations and cannot capture the effect of tariffs on the prosumer's optimal decisions. In the following subsection we show how the MUFs calculated by our approach can be linearized and used in a $[\mathrm{P} 2 \mathrm{P}$ market.

\section{Linear fit of the derived MUFs}

In most cases our proposed method reveals non-linear MUFs An example of a non-linear MUF is shown with blue color in Fig 10 However, linear functions have very attractive mathematical properties when it comes to negotiation processes and work well in decentralized or distributed optimization frameworks, which are mostly used in $\mathrm{P} 2 \mathrm{P}$ markets. It is thus useful to retrieve a good linear approximation of the revealed MUF to express prosumer preferences as accurately as possible. Without the proposed method, which incorporates a look-ahead strategy and considers all aspects of the prosumer optimization problem, the MUF is typically constructed based on an arbitrary methodology. Such a standard method from the literature was presented in the previous subsection and most often results in a very different MUF, as shown with green color in Fig. 10 (benchmark function). It is thus necessary to first reveal the actual MUF (which is generally non-linear), as done with the proposed approach, and then linearize it. This can be done by a least-squares fit so that the resulting function has the shape of Fig. 1. The accuracy of the approximation depends on the actual MUF, but the described two-step process will produce a linear MUF which most closely expresses the true prosumer preferences. In many cases, as the one in Fig. 10. the fitted function will be very similar to the revealed one, in contrast to the benchmark function.

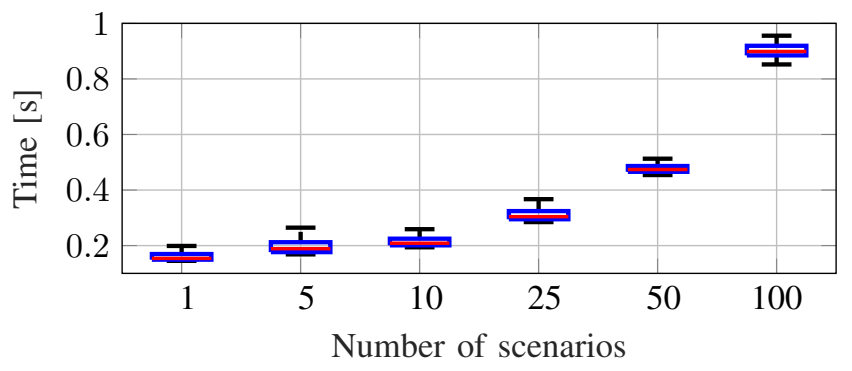

Fig. 11: Boxplots of the required computational time per execution for different numbers of used scenarios.

\section{E. Computational time}

The computational requirements of the proposed method are fairly low and can be carried out by a prosumer's EMS For the optimization problems MATLAB and the YALMIP frame [37] were used, with Gurobi 9.0 [38] as the solver. In Fig. 11 the computational times per execution are reported for an optimization horizon of 48 steps and different numbers of scenarios. Even when the full uncertainty set $\mathcal{W}$ is used, it takes less than one second per execution to produce one point in the MUF 15 points are sufficient to construct a function with a reasonable granularity, which means that the function can be constructed in less than 15 seconds.

\section{CONCLUSION}

The literature commonly adopts linear MUFs for representing the prosumers' willingness to trade energy, due to their attractive properties in distributed optimization frameworks. In this paper we investigated how actual MUFs look like, by proposing an $\mathrm{SO}$ approach that models the prosumer's decision-making process. This allows us to derive such functions without predefining their shape or arbitrarily choosing their parameters when assuming a linear form. The proposed method is generic to include any form of tariffs, flexible asset type or scenario generation technique.

Our results showed that actual MUFs have often non-linear properties and are primarily influenced by the presence of tariffs, the initial $\mathrm{SOC}$ and time of day. We further showed that MUFs can be relatively flat or even insensitive to large price ranges, while they may occasionally present large rates of change. This results in non-smooth shapes of the MUFs in certain cases. Even when the actual MUF has a linear shape, as assumed by the literature, the exact parameters are hard to tune without an approach that reveals them under conditions such as the uncertainty distribution, tariffs, and the prosumer's energy management strategy.

P2P markets most often require that prosumers use linear MUFs especially in cases where distributed optimization frameworks are applied, for computational reasons and in order to guarantee convergence to the globally optimal solution. Recognizing this requirement, we further proposed to apply a least-squares estimator to obtain linearized MUFs from our proposed method. These approximated functions guarantee the linear shape while at the same time they are 
a close approximation of the true preferences of the prosumer. By using MUFs that express true utility, instead of using an artificial representation, prosumers can interact with other agents while being aware of the true economic and social benefits of trading. As future work we plan to analyse the effect of using the proposed methodology in a large case study that simulates a realistic $\mathrm{P} 2 \mathrm{P}$ negotiation process.

\section{ACKNOWLEDGEMENT}

This work is partly supported by the Danish ForskEL and EUDP programmes through the Energy Collective Project (Grant no. 2016-1-12530).

\section{REFERENCES}

[1] F. F. Wu and P. Varaiya, "Coordinated multilateral trades for electric power networks: Theory and implementation," International Journal of Electrical Power \& Energy Systems, vol. 21, no. 2, pp. 75 - 102, 1999.

[2] H. Beitollahi and G. Deconinck, "Peer-to-peer networks applied to power grid," in Proceedings of the International conference on Risks and Security of Internet and Systems (CRiSIS), 2007.

[3] B. Cornélusse, I. Savelli, S. Paoletti, A. Giannitrapani, and A. Vicino, "A community microgrid architecture with an internal local market," Applied Energy, vol. 242, pp. 547 - 560, 2019.

[4] K. Zhang, S. Troitzsch, S. Hanif, and T. Hamacher, "Coordinated market design for peer-to-peer energy trade and ancillary services in distribution grids," IEEE Transactions on Smart Grid, vol. 11, no. 4, pp. 2929-2941, 2020.

[5] "Optimal collaborative demand-response planner for smart residential buildings," Energy, vol. 161, pp. 370 - 380, 2018.

[6] Y. Parag and B. K. Sovacool, "Electricity market design for the prosumer era," Nature energy, vol. 1, no. 16032, 2016.

[7] T. Morstyn, N. Farrell, S. J. Darby, and M. D. McCulloch, "Using peer-to-peer energy-trading platforms to incentivize prosumers to form federated power plants," Nature Energy, vol. 3, no. 2, pp. 94-101, 2018.

[8] T. Sousa, T. Soares, P. Pinson, F. Moret, T. Baroche, and E. Sorin, "Peer-to-peer and community-based markets: A comprehensive review," Renewable and Sustainable Energy Reviews, vol. 104, pp. 367 - 378, 2019.

[9] L. Einav, C. Farronato, and J. Levin, "Peer-to-peer markets," Annual Review of Economics, vol. 8, no. 1, pp. 615-635, 2016.

[10] Eurelectric, Prosumers - an integral part of the power system and the market. June 2015 (Accessed on Mar 2021).

[11] D. Peng and R. Poudineh, Electricity market design for a decarbonised future: An integrated approach. The Oxford Institute for Energy Studies, University of Oxford, 2017.

[12] European commission, Directive (EU) 2018/2001 of the European parliament and of the council of 11 December 2018 on the promotion of the use of energy from renewables. 2018 (Accessed on Mar 2021).

[13] D. K. Gode and S. Sunder, "Allocative efficiency of markets with zero-intelligence traders: Market as a partial substitute for individual rationality," Journal of Political Economy, vol. 101, no. 1, pp. 119-37, 1993.

[14] T. Morstyn, A. Teytelboym, and M. McCulloch, "Designing decentralized markets for distribution system flexibility," IEEE Transactions on Power Systems, vol. 34, no. 3, pp. 2128-2139, 2018.

[15] F. Moret and P. Pinson, "Energy collectives: A community and fairness based approach to future electricity markets," IEEE Transactions on Power Systems, vol. 34, no. 5, pp. 3994-4004, 2019.

[16] W. El-Baz, P. Tzscheutschler, and U. Wagner, "Integration of energy markets in microgrids: A double-sided auction with device-oriented bidding strategies," Applied Energy, vol. 241, pp. 625 - 639, 2019.

[17] E. Sorin, L. Bobo, and P. Pinson, "Consensus-based approach to peer-topeer electricity markets with product differentiation," IEEE Transactions on Power Systems, vol. 34, no. 2, pp. 994-1004, 2019.

[18] T. Baroche, P. Pinson, R. L. G. Latimier, and H. Ben Ahmed, "Exogenous cost allocation in peer-to-peer electricity markets," IEEE Transactions on Power Systems, vol. 34, no. 4, pp. 2553-2564, 2019.

[19] M. Fahrioglu and F. L. Alvarado, "Using utility information to calibrate customer demand management behavior models," IEEE Transactions on Power Systems, vol. 16, no. 2, pp. 317-322, 2001.
[20] P. Samadi, A. Mohsenian-Rad, R. Schober, V. W. S. Wong, and J. Jatskevich, "Optimal real-time pricing algorithm based on utility maximization for smart grid," in 2010 First IEEE International Conference on Smart Grid Communications, pp. 415-420, 2010.

[21] G. Hug, S. Kar, and C. Wu, "Consensus + innovations approach for distributed multiagent coordination in a microgrid," IEEE Transactions on Smart Grid, vol. 6, no. 4, pp. 1893-1903, 2015.

[22] A. Paudel, K. Chaudhari, C. Long, and H. B. Gooi, "Peer-to-peer energy trading in a prosumer-based community microgrid: A game-theoretic model," IEEE Transactions on Industrial Electronics, vol. 66, no. 8, pp. 6087-6097, 2019.

[23] A. Paudel, L. P. M. I. Sampath, J. Yang, and H. B. Gooi, "Peer-to-peer energy trading in smart grid considering power losses and network fees," IEEE Transactions on Smart Grid, vol. 11, no. 6, pp. 4727-4737, 2020.

[24] W. Tushar, B. Chai, C. Yuen, D. B. Smith, K. L. Wood, Z. Yang, and H. V. Poor, "Three-party energy management with distributed energy resources in smart grid," IEEE Transactions on Industrial Electronics, vol. 62, no. 4, pp. 2487-2498, 2015.

[25] S. Maharjan, Q. Zhu, Y. Zhang, S. Gjessing, and T. Başsar, "Dependable demand response management in the smart grid: A stackelberg game approach," IEEE Transactions on Smart Grid, vol. 4, no. 1, pp. 120132,2013

[26] N. Liu, X. Yu, C. Wang, and J. Wang, "Energy sharing management for microgrids with PV prosumers: A Stackelberg game approach," IEEE Transactions on Industrial Informatics, vol. 13, no. 3, pp. 1088-1098, 2017.

[27] T. Baroche, F. Moret, and P. Pinson, "Prosumer markets: A unified formulation," in 2019 IEEE Milan PowerTech, pp. 1-6, 2019.

[28] A. Mas-Colell, M. D. Whinston, and J. R. Green, Microeconomic Theory. No. 9780195102680 in OUP Catalogue, Oxford University Press, 1995.

[29] "Powerpeers [online].” Available: https://www.powerpeers.nl// Accessed on Mar 2021.

[30] "lo3energy [online]." Available: https://lo3energy.com/ Accessed on Mar 2021.

[31] E. McKenna and M. Thomson, "Photovoltaic metering configurations, feed-in tariffs and the variable effective electricity prices that result," IET Renewable Power Generation, vol. 7, no. 3, pp. 235-245, 2013.

[32] I. Savelli, A. De Paola, and F. Li, "Ex-ante dynamic network tariffs for transmission cost recovery," Applied Energy, vol. 258, p. 113979, 2020.

[33] E. Mengelkamp, J. Gärttner, K. Rock, S. Kessler, L. Orsini, and C. Weinhardt, "Designing microgrid energy markets: A case study: The brooklyn microgrid," Applied Energy, vol. 105, pp. 870-880, 2017.

[34] T. Morstyn, A. Teytelboym, and M. D. McCulloch, "Bilateral contract networks for peer-to-peer energy trading," IEEE Transactions on Smart Grid, vol. 10, no. 2, pp. 2026-2035, 2019.

[35] J. Fortuny-Amat and B. McCarl, "A representation and economic interpretation of a two-level programming problem," Journal of the Operational Research Society, vol. 32, no. 9, pp. 783-792, 1981.

[36] E. L. Ratnam, S. R. Weller, C. M. Kellett, and A. T. Murray, "Residential load and rooftop PV generation: an Australian distribution network dataset," International Journal of Sustainable Energy, vol. 36, no. 8, pp. 787-806, 2017.

[37] J. Lofberg, "Yalmip: A toolbox for modeling and optimization in matlab," 2004

[38] L. Gurobi Optimization, "Gurobi optimizer reference manual," 2021.

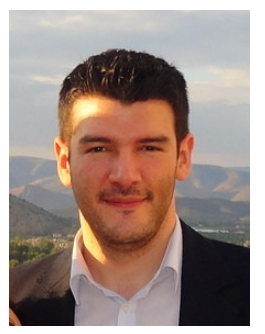

Charalampos Ziras (M'19) received the Dipl.-Ing degree in electrical and computer engineering from the National Technical University of Athens, Athens, Greece, and the M.Sc. degree in energy science and technology from ETH Zurich (Swiss Federal Institute of Technology), Zurich, Switzerland. He obtained the Ph.D. degree in power systems from the Center for Electric Power and Energy, Technical University of Denmark (DTU), Lyngby, Denmark, where he is currently working as a Post-Doc. His research interests include aggregation, optimization, and control of DERs, as well as DSO mechanisms for the integration of large shares of DERs in distribution networks. 


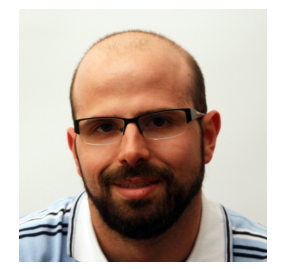

Tiago Sousa (M'19) received the M.Sc. degree in Electrical Engineering from the Polytechnic Institute of Porto (ISEP/IPP), Porto, Portugal in 2011, and the Ph.D. degree in Electrical and Computer Science Engineering from Instituto Superior Técnico (IST) at University of Lisbon, Lisbon, Portugal. He is Post-doc with the Technical University of Denmark, Department of Electrical Engineering, Center for Electric Power and Energy. His research interests include distributed optimization for energy markets, peer-to-peer trading and communication, artificial intelligence applied to scheduling problems, and reinforcement learning.

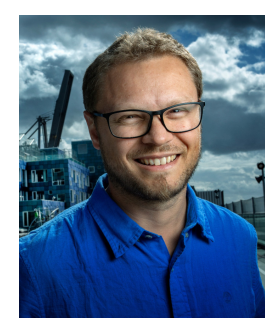

Pierre Pinson (S'13, F'20) received the M.Sc degree in applied mathematics from the National Institute for Applied Sciences, Toulouse, France, and the Ph.D. degree in energetics from Ecole des Mines de Paris, Paris, France. He is a Professor with the Technical University of Denmark, Department of Technology, Management and Economics. His research interests include forecasting, uncertainty estimation, optimization under uncertainty, decision sciences, and renewable energies. He is the Editorin-Chief for the International Journal of Forecasting. 